PROCEEDINGS OF THE

AMERICAN MATHEMATICAL SOCIETY

Volume 139, Number 12, December 2011, Pages 4445-4459

S 0002-9939(2011)10915-8

Article electronically published on April 5, 2011

\title{
PLANAR LOOPS WITH PRESCRIBED CURVATURE: EXISTENCE, MULTIPLICITY AND UNIQUENESS RESULTS
}

\author{
ROBERTA MUSINA
}

(Communicated by Matthew J. Gursky)

\begin{abstract}
Let $k: \mathbb{C} \rightarrow \mathbb{R}$ be a smooth given function. A $k$-loop is a closed curve $u$ in $\mathbb{C}$ having prescribed curvature $k(p)$ at every point $p \in u$. We use variational methods to provide sufficient conditions for the existence of $k$-loops. Then we show that a breaking symmetry phenomenon may produce multiple $k$-loops, in particular when $k$ is radially symmetric and somewhere increasing. If $k>0$ is radially symmetric and non-increasing, we prove that any embedded $k$-loop is a circle; that is, round circles are the only convex loops in $\mathbb{C}$ whose curvature is a non-increasing function of the Euclidean distance from a fixed point. The result is sharp, as there exist radially increasing curvatures $k>0$ which have embedded $k$-loops that are not circles.
\end{abstract}

\section{INTRODUCTION}

In this paper we prove existence, multiplicity and uniqueness results for the following $k$-loop problem: given a non constant function $k: \mathbb{C} \rightarrow \mathbb{R}$, find a $k$-loop, that is, a closed curve $u$ in $\mathbb{C}$ having prescribed curvature $k(p)$ at every point $p \in u$.

Several papers deal with loops with prescribed curvature and with related questions; see for example [4, [5], 8], 9], [12, [13]. For similar problems in higher dimensions we quote [3, 6], 7], 13] and references therein.

In Section 2 we provide global sufficient conditions for the existence of $k$-loops by studying the ordinary differential system

$$
\left\{\begin{array}{l}
u^{\prime \prime}=\left(\int_{0}^{1}\left|u^{\prime}\right|^{2}\right)^{1 / 2} k(u)\left(i u^{\prime}\right) \quad \text { on }(0,1), \\
u \in H_{\text {per }}
\end{array}\right.
$$

where $H_{\text {per }}$ is the Sobolev space of 1 -periodic functions in $H_{\text {loc }}^{1}(\mathbb{R}, \mathbb{C})$. Any non constant weak solution $u$ to (0.1) is smooth by regularity theory. As $u^{\prime \prime}$ is orthogonal to $u^{\prime}$, we have that $\left|u^{\prime}\right|$ is a constant. Thus $u^{\prime \prime} \cdot\left(i u^{\prime}\right)=\left|u^{\prime}\right|^{3} k(u)$; that is, $u$ is a $k$-loop.

If the prescribed curvature $k \in C^{2}(\mathbb{C})$ satisfies

$\left(\mathrm{k}_{1}\right) M_{k}:=\sup _{z \in \mathbb{C}}|(\nabla k(z) \cdot z) z|<1$,

$\left(\mathrm{k}_{2}\right)$ there exists a constant $k_{\infty}>0$ such that $k(z)=k_{\infty}+o\left(|z|^{-1}\right)$ as $|z| \rightarrow \infty$, then problem (0.1) can be plainly studied via variational methods. Indeed solutions to (10.1) turn out to be critical points of an energy functional $\mathcal{E}_{k}: H_{\text {per }} \rightarrow \mathbb{R}$ that

Received by the editors May 3, 2010 and, in revised form, October 14, 2010.

2010 Mathematics Subject Classification. Primary 51M25, 53A04, 49J10.

(C)2011 American Mathematical Society Reverts to public domain 28 years from publication 4445 
enjoys good regularity and geometrical properties thanks to assumptions $\left(\mathrm{k}_{1}\right)$ and $\left(\mathrm{k}_{2}\right)$ (see Section 1 for details). In particular the Nehari manifold

$$
\Sigma=\left\{u \in H_{\text {per }} \mid u \text { is non-constant, } \mathcal{E}_{k}^{\prime}(u) \cdot u=0\right\}
$$

is a smooth submanifold of $H_{p e r}$ and the functional $\mathcal{E}_{k}$ is positively bounded from below on $\Sigma$. Notice that every $k$-loop admits a parametrization $u \in \Sigma$. In spite of the non-homogeneous nature of problem (0.1), it turns out that $\Sigma$ is a natural constraint for $\mathcal{E}_{k}$; that is, any stationary point for $\mathcal{E}_{k}(u)$ on $\Sigma$ is a $k$-loop. In particular, if $u \in \Sigma$ achieves the infimum

$$
\underline{c}:=\inf _{u \in \Sigma} \mathcal{E}_{k}(u),
$$

then $u$ is a minimal $k$-loop, that means that $u$ has the minimal energy among all $k$-loops.

The infimum $\underline{c}$ might be not achieved, due to the lack of compactness produced by the group of translations $u \mapsto u+p$. On the other hand, in Lemma 1.4 we show that $\underline{c} \leq \pi / k_{\infty}$. Then we prove in Theorem 2.1 that a minimal $k$-loop $u \in \Sigma$ exists, provided that $\underline{c}<\pi / k_{\infty}$. In particular, it turns out that $\underline{c}<\pi / k_{\infty}$ if $k(z)>k_{\infty}$ for $|z|$ large; see Theorem [2.5]

The main results in the present paper highlight the connection between the monotonicity properties of the prescribed curvature $k$ along radial directions and the existence of geometrically distinct $k$-loops.

For the sake of clarity in this introduction we restrict ourselves to radially symmetric curvatures. It is straightforward to notice that if $k(z)=k(|z|)$, then $\mathcal{R} \circ u$ is a $k$-loop for any $k$-loop $u$ and for any rotation $\mathcal{R}$ of the complex plane. Thus, any non-round $k$-loop generates a 1 -dimensional manifold of distinct $k$-loops.

Multiple $k$-loops may be produced by a breaking symmetry phenomenon for problem (0.2). For instance, one can construct a curvature $k(z)=k(|z|)$ satisfying $\left(\mathrm{k}_{1}\right),\left(\mathrm{k}_{2}\right)$ and such that there exist a $k$-loop which is a circle $C_{R}$ about the origin and a minimal $k$-loop which is not a circle (see Corollary 3.2). Here we have parametrized $C_{R}$ by the map $t \mapsto R e^{2 \pi i t}$, so that $C_{R}$ can be regarded as a $k$-loop in $H_{p e r}$. More precisely, it turns out that $C_{R}$ is not a minimal $k$-loop if $k$ increases along the normal directions to $C_{R}$.

Theorem 0.1. Assume that $k \in C^{2}(\mathbb{C})$ is a radially symmetric curvature satisfying $\left(\mathrm{k}_{1}\right)$ and $\left(\mathrm{k}_{2}\right)$. If there is a radius $R>0$ with $R k(R)=1$ and $k^{\prime}(R)>0$, then the circle $C_{R}$ is not a minimal $k$-loop.

Theorem 0.1 is a consequence of a more general result that will be stated in Section 3 (see Theorem 3.1).

Roughly speaking, if the prescribed curvature is somewhere increasing, then one can expect breaking symmetry phenomena and multiple $k$-loops. This might happen also for curvatures that do not satisfy the assumptions $\left(\mathrm{k}_{1}\right)$ and $\left(\mathrm{k}_{2}\right)$. In Example 4.2 we notice that the curvature of any ellipse increases with the distance from the center. Conversely, non-increasing curvatures may have only round $k$ loops.

Theorem 0.2. Let $k \in C^{0}(\mathbb{C})$ be a positive and radially symmetric function. Assume that $k$ is non-increasing as a function of the distance from the origin. Then any embedded $k$-loop is a circle.

The result is sharp, in view of Example 4.2 in Section 4. 
Our proof of Theorem 0.2 is based on Osserman's construction for the four-vertex theorem. One of the main tools is the Touching Lemma for the mean curvature operator, that follows from Hopf's maximum principle.

Several uniqueness (up to homothety) results are available for similar geometrical problems. A complete list of references would lead us far from our purpose. We quote the pioneering papers 2 by Alexandrov and 1 by Aeppli, where the prescribed curvature is assumed to be homogeneous of degree -1 . Treibergs and Wei 13 proved the uniqueness of embedded radial graphs over the unit sphere $\mathbb{S}^{n}$ having positive prescribed mean curvature $H \in C^{1}\left(\mathbb{R}^{n+1}\right)$, such that $H(p)+\nabla H(p) \cdot p \leq 0$ for any $p \in \mathbb{R}^{n+1}$.

A result similar to Theorem 0.2 is missing for the $H$-bubble problem. It would be of interest to know if spheres are the only convex surfaces in $\mathbb{R}^{3}$ whose mean curvature $H$ is a non-increasing function of the distance from the origin.

Notation. Let $z_{1}, z_{2}$ be two points in the complex plane $\mathbb{C}$. We denote by $z_{1} z_{2} \in \mathbb{C}$ and by $z_{1} \cdot z_{2} \in \mathbb{R}$ their complex and scalar products, respectively. Let $R>0$ and $z \in \mathbb{C}$. The open disk of center $z$ and radius $R$ is denoted by $D_{R}(z)$. If $z=0$ we simply write $D_{R}$ instead of $D_{R}(0)$. The unit circle $\mathbb{S}^{1}=\partial D_{1}$ is parametrized by the function

$$
\omega(t):=e^{2 \pi i t}, \quad \omega:(0,1) \rightarrow \mathbb{C} .
$$

A loop is a closed curve parametrized by a periodic function $g: \mathbb{R} \rightarrow \mathbb{C}$ of class $C^{2}$ and such that $g^{\prime}(t) \neq 0$ for any $t \in I$. We will often identify the curve $g$ with its image. It is well known that the only loops having constant curvature are circles. An embedded loop is a closed curve without self-intersections. Any compact, connected, 1-dimensional submanifold of $\mathbb{C}$ without boundary is an embedded loop.

We let

$$
H_{\text {per }}=\left\{\bar{u} \circ \omega \mid \bar{u} \in H^{1}\left(\mathbb{S}^{1}, \mathbb{C}\right)\right\},
$$

where $\omega$ is defined in (0.3). We define in $H_{\text {per }}$ the Hilbertian scalar product

$$
\langle u, v\rangle=\int_{0}^{1} u^{\prime} v^{\prime}+\left(\int_{0}^{1} u\right) \cdot\left(\int_{0}^{1} v\right) .
$$

Notice that $H_{\text {per }}$ contains $\mathbb{C}$ as a closed subspace. We denote by $H_{p e r}^{-1}$ the topological dual space to $H_{p e r}$.

\section{Preliminaries}

We start by recalling the main features of the variational approach to problem (0.1). For details we refer to [5] and to [9]. Let $k \in C^{0}(\mathbb{C})$ be a given bounded function, and set

$$
m(z)=\int_{0}^{1} k(s z) s d s, \quad m: \mathbb{C} \rightarrow \mathbb{R}
$$

For $u \in H_{p e r}$ we put

$$
\mathcal{L}(u)=\left(\int_{0}^{1}\left|u^{\prime}\right|^{2}\right)^{1 / 2}, \quad \mathcal{A}_{k}(u)=\int_{0}^{1} m(u) u \cdot\left(i u^{\prime}\right) .
$$

The functional $\mathcal{A}_{k}(u)$ is well defined on $H_{\text {per }}$ as $u \in L^{\infty}$ for any $u \in H_{\text {per }}$, by the Sobolev embedding theorem. The real number $\mathcal{A}_{k}(u)$ measures the algebraic area 
enclosed by the curve $u$ with respect to the weight $k$. Moreover, the following isoperimetric inequality holds:

$$
4 \pi\left|\mathcal{A}_{k}(u)\right| \leq\|k\|_{\infty} \mathcal{L}(u)^{2} \quad \text { for any } u \in H_{\text {per }} .
$$

For any constant $k_{\infty} \neq 0$ it turns out that

$$
\mathcal{A}_{k_{\infty}}(u)=\frac{k_{\infty}}{2} \int_{0}^{1} u \cdot\left(i u^{\prime}\right) .
$$

In the next lemma we state some remarks on the area functional. We omit the simple proofs.

Lemma 1.1. Let $k \in C^{1}(\mathbb{C})$. Assume that $M_{k}=\sup _{z \in \mathbb{C}}|(\nabla k(z) \cdot z) z|$ is finite and that there exists $\lim _{|z| \rightarrow \infty} k(z)=k_{\infty} \in \mathbb{R}$. Then

$$
\begin{gathered}
\left|\left(k(z)-k_{\infty}\right) z\right| \leq M_{k} \\
|(2 m(z)-k(z)) z| \leq M_{k}, \\
2\left|\mathcal{A}_{k-k_{\infty}}(u)\right| \leq M_{k} \mathcal{L}(u) \quad \text { for any } u \in H_{p e r} .
\end{gathered}
$$

The energy $\mathcal{E}_{k}: H_{\text {per }} \rightarrow \mathbb{R}$ is defined by

$$
\mathcal{E}_{k}(u)=\mathcal{L}(u)+\mathcal{A}_{k}(u)=\left(\int_{0}^{1}\left|u^{\prime}\right|^{2}\right)^{1 / 2}+\int_{0}^{1} m(u) u \cdot\left(i u^{\prime}\right) .
$$

If $k$ is of class $C^{1}$, then the functional $\mathcal{E}_{k}$ is Frechét differentiable on $H_{p e r} \backslash \mathbb{C}$ and

$$
\mathcal{E}_{k}^{\prime}(u) \cdot \varphi=\frac{1}{\mathcal{L}(u)} \int_{0}^{1} u^{\prime} \cdot \varphi^{\prime}+\int_{0}^{1} k(u) \varphi \cdot\left(i u^{\prime}\right) \quad \text { for any } u \in H_{p e r} \backslash \mathbb{C}, \varphi \in H_{p e r}
$$

(see for example [5]). In particular, any critical point for $\mathcal{E}_{k}$ on $H_{\text {per }} \backslash \mathbb{C}$ parametrizes a smooth $k$-loop.

Remark 1.2. Let $k$ be as in Lemma 1.1 and assume in addition that $k_{\infty} \neq 0$. Then the energy $\mathcal{E}_{k}$ is unbounded from below. Fix any map $u \in H_{\text {per }}$ such that $\mathcal{A}_{k_{\infty}}(u)<0$. Then, using (1.5), we get

$$
\begin{aligned}
\mathcal{E}_{k}(s u) & =\mathcal{L}(s u)+\mathcal{A}_{k-k_{\infty}}(s u)+\mathcal{A}_{k_{\infty}}(u) \\
& \leq\left(1+M_{k}\right) s \mathcal{L}(u)+s^{2} \mathcal{A}_{k_{\infty}}(u) \rightarrow-\infty \quad \text { as } s \rightarrow \infty .
\end{aligned}
$$

Next we introduce the curvature function

$$
f(z):=2 k(z)+(\nabla k(z) \cdot z), \quad f: \mathbb{C} \rightarrow \mathbb{R} .
$$

Since $\int_{0}^{1} f(s z) s d s=k(z)$, then from (1.6) we infer

$$
\mathcal{E}_{k}^{\prime}(u) \cdot u=\mathcal{L}(u)+\int_{0}^{1} k(u) u \cdot\left(i u^{\prime}\right)=\mathcal{E}_{f}(u) .
$$

Thus, $\mathcal{E}_{k}^{\prime}(u) \cdot u$ equals the energy of $u$ with respect to the curvature $f$. If $k$ is of class $C^{2}$, then, coherently with (1.6), the functional $\mathcal{E}_{f}$ is Frechét differentiable on $H_{\text {per }} \backslash \mathbb{C}$, and

$$
\mathcal{E}_{f}^{\prime}(u) \cdot \varphi=\frac{1}{\mathcal{L}(u)} \int_{0}^{1} u^{\prime} \cdot \varphi^{\prime}+\int_{0}^{1}(2 k(u)+(\nabla k(u) \cdot u)) \varphi \cdot\left(i u^{\prime}\right)
$$

for any $u \in H_{\text {per }} \backslash \mathbb{C}, \varphi \in H_{\text {per }}$. Lastly we introduce the Nehari manifold

$$
\Sigma=\left\{u \in H_{\text {per }} \mid \mathcal{L}(u)>0, \mathcal{E}_{k}^{\prime}(u) \cdot u=\mathcal{E}_{f}(u)=0\right\}
$$


and the infimum

$$
\underline{c}:=\inf _{\Sigma} \mathcal{E}_{k} .
$$

Remark 1.3. Assume that $k \in C^{2}(\mathbb{C})$ satisfies $M_{k}<1$ and $k(z) \rightarrow k_{\infty}=0$ as $|z| \rightarrow \infty$. Then from (1.5), (1.7) and (1.3) it easily follows that the energy is coercive with respect to the seminorm $\mathcal{L}(u)$ and that the manifold $\Sigma$ is empty.

From now on we assume that $k \in C^{2}(\mathbb{C})$ satisfies the assumptions $\left(\mathrm{k}_{1}\right)$ and $\left(\mathrm{k}_{2}\right)$ in the introduction. In the next lemma we show that the set $\Sigma$ is not empty and smooth, and it is a natural constraint for $\mathcal{E}_{k}$.

Lemma 1.4. The following facts hold.

(1) Let $u \in H_{\text {per }} \backslash \mathbb{C}$. Then $u \in \Sigma$ if and only if $\mathcal{E}_{k}(u)=\sup _{s>0} \mathcal{E}_{k}(s u)$.

(2) $\Sigma$ is a non-empty submanifold of $H_{\text {per }}$ of class $C^{1}$.

(3) $0<\underline{c} \leq \pi / k_{\infty}$.

(4) Every critical point $u$ for $\mathcal{E}_{k}$ on $\Sigma$ solves $\mathcal{E}_{k}^{\prime}(u)=0$.

Proof. We start by noticing that

$$
\mathcal{E}_{f}^{\prime}(u) \cdot u=-\mathcal{L}(u)+\int_{0}^{1}(\nabla k(u) \cdot u) u \cdot\left(i u^{\prime}\right) \quad \text { for any } u \in \Sigma
$$

by (1.8). Thus from $\left(\mathrm{k}_{1}\right)$ we readily get

$$
-\left(1+M_{k}\right) \mathcal{L}(u) \leq \mathcal{E}_{f}^{\prime}(u) \cdot u \leq\left(-1+M_{k}\right) \mathcal{L}(u)<0 \quad \text { for any } u \in \Sigma .
$$

To prove (1) we fix any function $u$ in $H_{p e r} \backslash \mathbb{C}$ such that $\mathcal{E}_{k}(u)=\sup _{s>0} \mathcal{E}_{k}(s u)$. Then the derivative of the function $s \mapsto \mathcal{E}_{k}(s u)$ vanishes at $s=1$; that is, $u \in \Sigma$. Conversely, if $u \in \Sigma$ we put $F(s):=\mathcal{E}_{k}(s u)$ for $s>0$. We use (1.7), (1.10) and (1.11) to get

$$
\begin{aligned}
s F^{\prime}(s) & =\mathcal{E}_{f}(s u), \\
s F^{\prime}(s)+s^{2} F^{\prime \prime}(s) & =\mathcal{E}_{f}^{\prime}(s u) \cdot(s u) \leq\left(-1+M_{k}\right) s^{2} \mathcal{L}(u) .
\end{aligned}
$$

In particular, $s=1$ is a critical point for $F$ on $\{s>0\}$ and every critical point for $F$ is a local maximum for $F$. Thus the function $F$ achieves its absolute maximum at $s=1$; that is, $\mathcal{E}_{k}(u)=\sup _{s>0} \mathcal{E}_{k}(s u)$. This completes the proof of (1).

We point out that from (1) and from Remark 1.2 it follows that $\Sigma$ is non-empty and

$$
\underline{c}=\inf _{\substack{u \in H_{p e r} \\ \mathcal{A}_{k_{\infty}}(u)<0}} \sup _{s>0} \mathcal{E}_{k}(s u) .
$$

Since $k \in C^{2}(\mathbb{C})$ we have that $\mathcal{E}_{f}$ is of class $C^{1}$ on $H_{\text {per }} \backslash \mathbb{C}$. In addition, from (1.11) it follows that $\mathcal{E}_{f}^{\prime}(u) \neq 0$ for any $u \in \Sigma$. Thus the constraint $\Sigma$ has a normal direction at every point, and claim (2) is proved.

To check that $\underline{c}$ is positive we use the isoperimetric inequality (1.1). For any $u \in \Sigma$ we get $0=\overline{\mathcal{E}}_{f}(u)=\mathcal{L}(u)+\mathcal{A}_{f}(u) \geq \mathcal{L}(u)-\|f\|_{\infty}(4 \pi)^{-1} \mathcal{L}(u)^{2}$. In particular,

$$
\|f\|_{\infty} \mathcal{L}(u) \geq 4 \pi \text { for any } u \in \Sigma \text {. }
$$

As $\mathcal{E}_{f}(u)=0$ for any $u \in \Sigma$, then from (1.7) we get

$$
2 \mathcal{E}_{k}(u)=\mathcal{L}(u)+\int_{0}^{1}(2 m(u)-k(u)) u \cdot\left(i u^{\prime}\right) \quad \text { for any } u \in \Sigma .
$$


Thus from (1.4) we infer

$$
2 \mathcal{E}_{k}(u) \geq\left(1-M_{k}\right) \mathcal{L}(u) \text { for any } u \in \Sigma,
$$

that compared with (1.13) gives $\underline{c}>0$, since $M_{k}<1$. To prove that $\underline{c} \leq \pi / k_{\infty}$ we parametrize the unit circle around 0 with the curve $\omega(t)=e^{2 \pi i t}$ as in (0.3). By (1.12) it turns out that

$$
\underline{c} \leq \sup _{s>0} \mathcal{E}_{k}(s(\omega+p))=\sup _{s>0}\left[\mathcal{E}_{k_{\infty}}(s(\omega+p))+\mathcal{A}_{k-k_{\infty}}(s(\omega+p))\right]
$$

for any $p \in \mathbb{C}$. It is easy to compute

$$
\mathcal{E}_{k_{\infty}}(s(\omega+p))=2 \pi s-\pi k_{\infty} s^{2} .
$$

Since $s(\omega+p)$ parametrizes the circle of radius $s$ around $s p$, then

$$
\left|\mathcal{A}_{k-k_{\infty}}(s(\omega+p))\right|=\left|\int_{D_{s}(s p)}\left(k(z)-k_{\infty}\right) d z\right| \leq M_{k} \int_{D_{s}(s p)} \frac{1}{|z|} d z
$$

by the divergence theorem and by (1.3). Thus for $|p|$ large it holds that

$$
\left|\mathcal{A}_{k-k_{\infty}}(s(\omega+p))\right| \leq M_{k} \int_{D_{s}(s p)} \frac{1}{s(|p|-1)} d z=M_{k} \frac{\pi s}{|p|-1},
$$

and therefore

$$
\sup _{s>0} \mathcal{E}_{k}(s(\omega+p)) \leq \sup _{s>0} \pi s\left(2+\frac{M_{k}}{|p|-1}-k_{\infty} s\right)=\frac{\pi}{k_{\infty}}+O\left(|p|^{-1}\right) .
$$

Hence $\underline{c} \leq \pi / k_{\infty}$, as desired.

It remains to prove (4). If $u \in \Sigma$ is a critical point for $\mathcal{E}_{k}$ on $\Sigma$, then there exists a Lagrange multiplier $\lambda \in \mathbb{R}$ such that $\mathcal{E}_{k}^{\prime}(u) \cdot \varphi=\lambda \mathcal{E}_{f}^{\prime}(u) \cdot \varphi$ for any $\varphi \in H_{\text {per }}$. In particular $\lambda \mathcal{E}_{f}^{\prime}(u) \cdot u=\mathcal{E}_{k}^{\prime}(u) \cdot u=0$ as $u \in \Sigma$. Thus $\lambda=0$ by (1.11), and $\mathcal{E}_{k}^{\prime}(u)=0$.

The next lemma will be crucial later on.

Lemma 1.5. For any $p \in \mathbb{C}$ there exists a unique $s_{p}>0$ such that $s_{p}(\omega+p) \in \Sigma$. Moreover, the function $p \mapsto s_{p}$ is of class $C^{1}$ on $\mathbb{C}$.

Proof. Argue as in the proof of claim (1) to check that for any $p \in \mathbb{C}$ the map $s \mapsto \mathcal{E}_{k}(s(\omega+p))$ achieves its maximum on $(0, \infty)$ at its unique critical point $s_{p} \in(0, \infty)$. Thus $s_{p}(\omega+p) \in \Sigma$. As $s_{p}$ is uniquely and implicitly defined by the equation $\mathcal{E}_{f}\left(s_{p}(\omega+p)\right)=0$, and since the functional $\mathcal{E}_{f}$ is continuously differentiable on $H_{p e r} \backslash \mathbb{C}$, then the function $p \mapsto s_{p}$ is of class $C^{1}$ on $\mathbb{C}$ by (1.11).

Remark 1.6. Lemma 1.4 holds whenever $M_{k}<1$ and $k(z) \rightarrow k_{\infty}>0$ as $|z| \rightarrow \infty$.

\section{EXISTENCE}

We are now in a position to prove our existence result.

Theorem 2.1. Assume that $k \in C^{2}(\mathbb{C})$ satisfies $\left(\mathrm{k}_{1}\right),\left(\mathrm{k}_{2}\right)$ and let $\underline{c}$ be the infimum in (1.9). If $\underline{c}<\pi / k_{\infty}$, then there exists a minimal $k$-loop. 
Proof. Let $u_{n} \in \Sigma$ be a minimizing sequence for $\mathcal{E}_{k}$ on $\Sigma$, that is,

$$
\mathcal{E}_{k}\left(u_{n}\right) \rightarrow \underline{c}<\frac{\pi}{k_{\infty}}
$$

By Ekeland's variational principle we can assume that $u_{n}$ is a sequence of approximate critical points for $\mathcal{E}_{k}$ on the constraint $\Sigma$. This means that

$$
\mathcal{E}_{k}^{\prime}\left(u_{n}\right)-\lambda_{n} \mathcal{E}_{f}^{\prime}\left(u_{n}\right) \rightarrow 0 \quad \text { in } H_{p e r}^{-1}
$$

for some $\lambda_{n} \in \mathbb{R}$. The seminorms $\mathcal{L}\left(u_{n}\right)$ are uniformly bounded by (1.15). Since the $L^{\infty}$ norm of $f(u)=2 k(u)+(\nabla k(u) \cdot u)$ is bounded by a constant that depends only on $k$, then the sequence $\mathcal{E}_{f}^{\prime}\left(u_{n}\right)$ is bounded in $H_{\text {per }}^{-1}$ by (1.8). Finally, we notice that the sequence $\mathcal{E}_{f}^{\prime}\left(u_{n}\right) \cdot u_{n}$ is bounded and bounded away from zero by (1.11).

To conclude, we only have to prove that the sequence

$$
p_{n}:=\int_{0}^{1} u_{n}
$$

is bounded in $\mathbb{C}$. Indeed, in this case we can assume that $u_{n} \rightarrow u$ weakly in $H_{\text {per }}$, for some $u \in H_{p e r}$. In particular, $u_{n} \rightarrow u$ uniformly on $[0,1]$ by the Sobolev embedding theorem. Test (2.2) with $u_{n}$ to get

$$
0=\mathcal{E}_{k}^{\prime}\left(u_{n}\right) \cdot u_{n}=\lambda_{n} \mathcal{E}_{f}^{\prime}\left(u_{n}\right) \cdot u_{n}+o(1) .
$$

Since the sequence $\mathcal{E}_{f}^{\prime}\left(u_{n}\right) \cdot u_{n}$ is bounded away from zero, then $\lambda_{n} \rightarrow 0$, which implies $\mathcal{E}_{k}^{\prime}\left(u_{n}\right) \rightarrow 0$ by (2.2). Since $\left|k\left(u_{n}\right)\left(u_{n}-u\right) \cdot\left(i u_{n}^{\prime}\right)\right| \leq\|k\|_{\infty}\left\|u_{n}-u\right\|_{\infty}\left|u_{n}^{\prime}\right| \rightarrow 0$ in $L^{1}$, then, in particular,

$o(1)=\mathcal{E}_{k}^{\prime}\left(u_{n}\right) \cdot\left(u_{n}-u\right)=\frac{1}{\mathcal{L}\left(u_{n}\right)} \int_{0}^{1} u_{n}^{\prime} \cdot\left(u_{n}^{\prime}-u^{\prime}\right)+o(1)=\frac{1}{\mathcal{L}\left(u_{n}\right)} \int_{0}^{1}\left|u_{n}^{\prime}-u^{\prime}\right|^{2}+o(1)$.

Taking (1.13) into account we infer that $u_{n}^{\prime} \rightarrow u^{\prime}$ strongly in $L^{2}$. Therefore $u_{n} \rightarrow u$ in the $H_{p e r}$-norm. Then $\mathcal{E}^{\prime}(u)=0$ and $\mathcal{E}_{k}(u)=\underline{c}$ easily follow by continuity.

Assume by contradiction that the averages $p_{n}$ are not bounded. Then, for a subsequence, it turns out that $u_{n}-p_{n} \rightarrow U$ weakly in $H_{p e r}$ and uniformly on $[0,1]$, where $U \in H_{\text {per }}$ has zero mean value on $(0,1)$. We can also assume that there exists

$$
\alpha:=\lim _{n \rightarrow \infty} \mathcal{L}\left(u_{n}\right) \in \mathbb{R} .
$$

Notice that $\alpha>0$ by (1.13). Since $u_{n}-p_{n} \rightarrow U$ and $\left|u_{n}\right| \rightarrow \infty$ uniformly, then from $\left(\mathrm{k}_{1}\right)$ and $\left(\mathrm{k}_{2}\right)$ it follows that

$$
\begin{gathered}
\left(k\left(u_{n}\right)-k_{\infty}\right) u_{n} \rightarrow 0 \quad \text { uniformly } \\
k\left(u_{n}\right)\left(u_{n}-p_{n}\right) \rightarrow k_{\infty} U \quad \text { uniformly } \\
\nabla k\left(u_{n}\right) \cdot u_{n} \rightarrow 0 \quad \text { uniformly }
\end{gathered}
$$

Using (2.3) we get

$$
\int_{0}^{1} k\left(u_{n}\right) u_{n} \cdot\left(i u_{n}^{\prime}\right)=k_{\infty} \int_{0}^{1} u_{n} \cdot\left(i u_{n}^{\prime}\right)+o(1)=k_{\infty} \int_{0}^{1} U \cdot\left(i U^{\prime}\right)+o(1),
$$

as $\int_{0}^{1}\left(i u_{n}^{\prime}\right)=0$. In particular, from $\mathcal{E}_{k}^{\prime}\left(u_{n}\right) \cdot u_{n}=0$ and from (1.7) we infer that $U \neq 0$ and

$$
\alpha=-k_{\infty} \int_{0}^{1} U \cdot\left(i U^{\prime}\right)
$$


Next we notice that $\left(k\left(u_{n}\right)-k_{\infty}\right) p_{n}=\left(k\left(u_{n}\right)-k_{\infty}\right)\left(p_{n}-u_{n}\right)+\left(k\left(u_{n}\right)-k_{\infty}\right) u_{n} \rightarrow 0$ uniformly, since $k\left(u_{n}\right) \rightarrow k_{\infty}$ uniformly, $\sup _{n}\left\|u_{n}-p_{n}\right\|<\infty$, and by (2.3). This implies

$$
\int_{0}^{1} k\left(u_{n}\right) p_{n} \cdot\left(i u_{n}^{\prime}\right)=k_{\infty} p_{n} \cdot \int_{0}^{1}\left(i u_{n}^{\prime}\right)+o(1)=o(1) .
$$

Using (1.6) once again we infer

$$
\mathcal{E}_{k}^{\prime}\left(u_{n}\right) \cdot\left(u_{n}-p_{n}\right)=-\mathcal{E}_{k}^{\prime}\left(u_{n}\right) \cdot p_{n}=\int_{0}^{1} k\left(u_{n}\right) p_{n} \cdot\left(i u_{n}^{\prime}\right)=o(1),
$$

as $u_{n} \in \Sigma$. By similar arguments, from (1.8) we also obtain

$$
\mathcal{E}_{f}^{\prime}\left(u_{n}\right) \cdot\left(u_{n}-p_{n}\right)=\alpha+2 k_{\infty} \int_{0}^{1} U \cdot\left(i U^{\prime}\right)+o(1)=-\alpha+o(1)
$$

by (2.4), (2.5) and (2.6). Consequently, since by (2.2) we have that

$$
\mathcal{E}_{k}^{\prime}\left(u_{n}\right) \cdot\left(u_{n}-p_{n}\right)=\lambda_{n} \mathcal{E}_{f}^{\prime}\left(u_{n}\right) \cdot\left(u_{n}-p_{n}\right)+o(1),
$$

then $o(1)=\lambda_{n}(-\alpha+o(1))$. Since $\alpha>0$, then $\lambda_{n} \rightarrow 0$. Thus $\mathcal{E}_{k}^{\prime}\left(u_{n}\right) \cdot \varphi \rightarrow 0$ for any $\varphi \in H_{\text {per }}$ by (2.2), as the sequence $\mathcal{E}_{f}^{\prime}\left(u_{n}\right)$ is bounded in $H_{p e r}^{-1}$. Therefore

$$
o(1)=\mathcal{E}_{k}^{\prime}\left(u_{n}\right) \cdot \varphi=\frac{1}{\alpha} \int_{0}^{1} U^{\prime} \varphi^{\prime}+2 k_{\infty} \int_{0}^{1} \varphi \cdot\left(i U^{\prime}\right)+o(1) \quad \text { for any } \varphi \in H_{p e r} .
$$

Thus, $U$ solves $U^{\prime \prime}=\alpha k_{\infty}\left(i U^{\prime}\right)$, and, in particular, $\mathcal{L}(U)=\alpha=\lim _{n} \mathcal{L}\left(u_{n}\right)$ by (2.6). This is sufficient to conclude that $u_{n}-p_{n} \rightarrow U$ strongly in $H_{p e r}$. In addition, $U$ is a non-constant solution to the linear ordinary differential system $U^{\prime \prime}=\mathcal{L}(U) k_{\infty}\left(i U^{\prime}\right)$. Thus $U$ parametrizes a circle of radius $k_{\infty}^{-1}$, and

$$
\mathcal{L}(U)=-k_{\infty} \int_{0}^{1} U \cdot\left(i U^{\prime}\right) \geq \frac{2 \pi}{k_{\infty}}, \quad \mathcal{E}_{k_{\infty}}(U)=\frac{L(U)}{2} .
$$

Next we claim that $\mathcal{E}_{k}\left(u_{n}\right)=\mathcal{E}_{k_{\infty}}(U)+o(1)$. Notice that

$$
\left(2 m\left(u_{n}\right)-k_{\infty}\right)\left(u_{n}\right)=2 \int_{0}^{1}\left(k\left(s u_{n}\right)-k_{\infty}\right)\left(s u_{n}\right) d s \rightarrow 0
$$

pointwise in $(0,1)$ by $\left(\mathrm{k}_{2}\right)$, (1.3) and Lebesgue's Theorem. Since, in addition, $i u_{n}^{\prime} \rightarrow i U^{\prime}$ in $L^{2}$, then

$$
2 \mathcal{A}_{k-k_{\infty}}\left(u_{n}\right)=\int_{0}^{1}\left(2 m\left(u_{n}\right)-k_{\infty}\right)\left(u_{n}\right) \cdot\left(i u_{n}^{\prime}\right)=o(1)
$$

by (1.4) and again by Lebesgue's Theorem. In conclusion, we get

$\mathcal{E}_{k}\left(u_{n}\right)=\mathcal{L}\left(u_{n}\right)+\frac{k_{\infty}}{2} \int_{0}^{1}\left(u_{n}-p_{n}\right) \cdot\left(i u_{n}^{\prime}\right)+\mathcal{A}_{k-k_{\infty}}\left(u_{n}\right)=\mathcal{E}_{k_{\infty}}(U)+o(1)=\frac{\pi}{k_{\infty}}+o(1)$.

Taking (2.1) and (2.8) into account we get

$$
\frac{\pi}{k_{\infty}}>\underline{c}=\mathcal{E}_{k}\left(u_{n}\right)+o(1)=\mathcal{E}_{k_{\infty}}(U)+o(1) \geq \frac{\pi}{k_{\infty}}+o(1),
$$

a contradiction for $n$ sufficiently large. This proves that the sequence $p_{n}$ is bounded in $\mathbb{C}$ and completes the proof of the theorem.

Remark 2.2. We notice that the assumption on the sign of $k_{\infty}$ is not restrictive. Indeed, $t \mapsto u(t)$ is a $k$-loop if and only if $t \mapsto u(1-t)$ has curvature $-k$. 
Remark 2.3. By suitably modifying the compactness argument in the proof of Theorem 2.1 one can completely describe the behavior of any sequence of approximate solutions. More precisely, assume that $u_{n} \in \Sigma$ satisfies $\mathcal{E}_{k}\left(u_{n}\right) \rightarrow c$ and $\mathcal{E}_{k}^{\prime}\left(u_{n}\right)-\lambda_{n} \mathcal{E}_{f}^{\prime}\left(u_{n}\right) \rightarrow 0$ for some $c \in \mathbb{R}$ and for a sequence $\lambda_{n} \in \mathbb{R}$. Then, up to a subsequence, either $u_{n}$ converges in $H_{p e r}$ to a $k$-loop $u$ such that $\mathcal{E}_{k}(u)=c$, or $\mu:=\pi c k_{\infty}^{-1}$ is a positive integer, and

$$
\left|\int_{0}^{1} u_{n}\right| \rightarrow \infty, \quad u_{n}-\int_{0}^{1} u_{n} \rightarrow U \text { in } H_{p e r},
$$

where $U(t)=k_{\infty}^{-1} \sigma e^{2 \pi \mu i t}$ for some $\sigma \in \mathbb{S}^{1}$.

Remark 2.4. Assume that $\underline{c}=\pi / k_{\infty}$ and that no minimal $k$-loop exists. Then using Remark 2.3 and arguing as in 7 one can find sufficient conditions for the existence of highly unstable $k$-loops.

Some conditions to ensure that $\underline{c}<\pi / k_{\infty}$ can be easily given. For instance, notice that

$$
\underline{c} \leq \sup _{s>0} \mathcal{E}_{k}(s(\omega+p))
$$

for any $p \in \mathbb{C}$, by (1.12), where $\omega$ is defined in (0.3). Since $s(\omega+p)$ parametrizes the circle of radius $s$ around $s p$, then $\mathcal{A}_{k}(s(\omega+p))=-\int_{D_{s}(s p)} k(z) d z$ by the divergence theorem. Thus

$$
\mathcal{E}_{k}(s(\omega+p))=2 \pi s-\int_{D_{s}(s p)} k(z) d z .
$$

In particular, $\underline{c}<\pi / k_{\infty}$ if there exists a point $p \in \mathbb{C}$ such that

$$
\sup _{s>0}\left(2 \pi s-\int_{D_{s}(s p)} k(z) d z\right)<\frac{\pi}{k_{\infty}} .
$$

This happens, for instance, if $k(z)>k_{\infty}$ on $\mathbb{C}$ (take $p=0$ ). Actually, weaker sufficient conditions can be given.

Theorem 2.5. Assume that $k \in C^{2}(\mathbb{C})$ satisfies $\left(\mathrm{k}_{1}\right)$, $\left(\mathrm{k}_{2}\right)$. If $k(z)>k_{\infty}$ for $|z|$ large, then there exists a minimal k-loop.

Proof. We only have to show that $\underline{c}<\pi / k_{\infty}$. Put as before $\omega(t)=e^{2 \pi i t}$. For any $p \in \mathbb{C}$ let $s_{p}>0$ be the unique positive number defined in Lemma 1.5. Then $\underline{c} \leq \mathcal{E}_{k}\left(s_{p}(\omega+p)\right)$, since $s_{p}(\omega+p) \in \Sigma$.

Recall from (1.7) that $\mathcal{E}_{f}(u)=\mathcal{L}(u)+k_{\infty} \int_{0}^{1} u \cdot\left(i u^{\prime}\right)+\int_{0}^{1}\left(k(u)-k_{\infty}\right) u \cdot\left(i u^{\prime}\right)$ for any $u \in H_{p e r}$, and therefore

$$
0=\mathcal{E}_{f}\left(s_{p}(\omega+p)\right)=2 \pi s_{p}-2 \pi k_{\infty} s_{p}^{2}+s_{p} \int_{0}^{1}\left(k\left(s_{p}(\omega+p)\right)-k_{\infty}\right) s_{p}(\omega+p) \cdot\left(i \omega^{\prime}\right) .
$$

In particular we infer

$$
k_{\infty} s_{p}=1-\int_{0}^{1}\left(k\left(s_{p}(\omega+p)\right)-k_{\infty}\right) s_{p}(\omega+p) \cdot \omega
$$

since $i \omega^{\prime}=-2 \pi \omega$. From (1.3) and (2.10) we get

$$
1-M_{k} \leq k_{\infty} s_{p} \leq 1+M_{k} \text { for any } p \in \mathbb{C} .
$$


Next we take a sequence of points $p_{n} \in \mathbb{C}$ such that $\left|p_{n}\right| \rightarrow \infty$. By (2.9), (2.11), and since $k(z)>k_{\infty}$ for $|z|$ large enough, we get

$$
\underline{c} \leq \mathcal{E}_{k}\left(s_{p_{n}}\left(\omega+p_{n}\right)\right)=2 \pi s_{p_{n}}-\int_{D_{s_{p_{n}}}\left(s_{p_{n}} p_{n}\right)} k(z) d z<2 \pi s_{p_{n}}-\pi k_{\infty} s_{p_{n}}^{2} .
$$

Thus $\underline{c}<\sup _{s>0}\left(2 \pi s-\pi k_{\infty} s^{2}\right)=\pi / k_{\infty}$, and the theorem is completely proved.

Remark 2.6. A slightly different proof can be obtained by following the arguments in [6], proof of Corollary 2.13. As in [6] the assumption on the sign of $k(z)-k_{\infty}$ can be weakened. It is sufficient to ask that there exist $\sigma \in \mathbb{S}^{1}, R, \delta>0$ such that $k(z)>k_{\infty}$ for any $z \in \mathbb{C} \backslash D_{R}$ with $\left.|\sigma-z| z\right|^{-1} \mid<\delta$.

\section{BREAKING SYMMETRY AND MULTIPLICITY}

In this section we identify the circle $C_{R}=\{|z|=R\}$ with its parametrization $t \mapsto R e^{2 \pi i t}$. With this notation we have that $C_{R}$ is a $k$-loop if and only if $R k(\cdot) \equiv 1$ on $C_{R}$. Theorem 0.1 is an immediate consequence of the next result.

Theorem 3.1. Let $k \in C^{2}(\mathbb{C})$ be a curvature satisfying $\left(\mathrm{k}_{1}\right)$ and $\left(\mathrm{k}_{2}\right)$. Assume that there exists $R>0$ such that $R k(z) \equiv 1$ and $\nabla k(z) \cdot z \geq 0$ for any $z \in C_{R}$. If the circle $C_{R}$ is a minimal $k$-loop, then $\nabla k(z) \cdot z \equiv 0$ on $C_{R}$.

Proof. We start by noticing that $u \in H_{p e r}$ is a $k$-loop if and only if $u_{R}:=R u$ is a

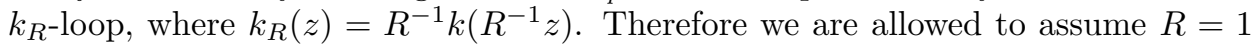
and $C_{R}=\mathbb{S}^{1}$. Hence $k \equiv 1$ on the unit sphere, $\mathbb{S}^{1}$ is a minimal $k$-loop and

$$
A(z):=\nabla k(z) \cdot z \geq 0 \quad \text { on } \mathbb{S}^{1} .
$$

Since $k \in C^{2}(\mathbb{C})$, then the energy $\mathcal{E}_{k}$ is twice differentiable on $H_{\text {per }} \backslash \mathbb{C}$, and

$$
\begin{aligned}
\mathcal{E}_{k}^{\prime \prime}(u)[\varphi, \psi]=\frac{1}{\mathcal{L}(u)} \int_{0}^{1} \varphi^{\prime} \cdot \psi^{\prime} & -\frac{1}{\mathcal{L}(u)^{3}}\left(\int_{0}^{1} u^{\prime} \cdot \varphi^{\prime}\right)\left(\int_{0}^{1} u^{\prime} \cdot \psi^{\prime}\right) \\
& +\int_{0}^{1} \varphi \cdot\left[k(u)\left(i \psi^{\prime}\right)+(\nabla k(u) \cdot \psi)\left(i u^{\prime}\right)\right]
\end{aligned}
$$

for all $u \in H_{p e r} \backslash \mathbb{C}$, and for any $\varphi, \psi \in H_{\text {per }}$ (see [5]). To compute $\mathcal{E}_{k}^{\prime \prime}(\omega)$ we notice that $\mathcal{L}(\omega)=2 \pi$ and $\omega^{\prime \prime}=2 \pi\left(i \omega^{\prime}\right)=-(2 \pi)^{2} \omega$. In particular $\int \omega^{\prime} \cdot \varphi^{\prime}=(2 \pi)^{2} \int \omega \cdot \varphi$ for any $\varphi \in H_{\text {per }}$. Since $k$ is constant on $\mathbb{S}^{1}$, then $\nabla k(\omega)=A(\omega) \omega$, where $A$ is defined in (3.1). Thus we get

$$
\begin{aligned}
\mathcal{E}_{k}^{\prime \prime}(\omega)[\varphi, \psi]=\frac{1}{2 \pi} \int_{0}^{1} \varphi^{\prime} \cdot \psi^{\prime} & -2 \pi\left(\int_{0}^{1} \omega \cdot \varphi\right)\left(\int_{0}^{1} \omega \cdot \psi\right) \\
& +\int_{0}^{1} \varphi \cdot\left[\left(i \psi^{\prime}\right)-2 \pi A(\omega)(\omega \cdot \psi) \omega\right]
\end{aligned}
$$

for any $\varphi, \psi \in H_{p e r}$. From (3.2) we first get $\mathcal{E}_{k}^{\prime \prime}(\omega)[\varphi, \omega]=-2 \pi \int_{0}^{1}(1+A(\omega)) \omega \cdot \varphi$ for any $\varphi \in H_{p e r}$, and in particular

$$
\mathcal{E}_{k}^{\prime \prime}(\omega)[\omega, \omega]=-2 \pi\left(1+\int_{0}^{1} A(\omega)\right)<0 .
$$

Taking instead $\varphi \equiv p$ to be a constant function we get

$$
\mathcal{E}_{k}^{\prime \prime}(\omega)[p, \omega]=-2 \pi \int_{0}^{1} A(\omega) \omega \cdot p,
$$


since $\omega$ has zero mean value on $(0,1)$. From (3.2) we infer also

$$
\mathcal{E}_{k}^{\prime \prime}(\omega)[p, p]=-2 \pi \int_{0}^{1} A(\omega)(\omega \cdot p)^{2}
$$

For any point $p \in \mathbb{C}$ we put $s(t):=s_{t p}$, where $s_{t p}>0$ is defined by the condition

$$
s_{t p}(\omega+t p)=s(t)(\omega+t p) \in \Sigma,
$$

as in Lemma 1.5. Since $\omega$ is a minimal $k$-loop, the function $g(t):=\mathcal{E}_{k}(s(t)(\omega+t p))$ attains its minimum at $t=0$. Notice that $s(0)=1$, since $\omega$ is a $k$-loop. Moreover the map $s(t)$ is of class $C^{1}$ on $(0, \infty)$ by Lemma 1.5 and

$$
\mathcal{E}_{k}^{\prime}(s(t)(\omega+t p)) \cdot(\omega+t p)=0 \quad \text { for any } t \in \mathbb{R} .
$$

We compute

$$
g^{\prime}(t)=\mathcal{E}_{k}^{\prime}(s(t)(\omega+t p)) \cdot\left(s^{\prime}(t)(\omega+t p)+s(t) p\right)=s(t) \mathcal{E}_{k}^{\prime}(s(t)(\omega+t p)) \cdot p,
$$

by (3.6). Thus $g$ is twice differentiable, and

$$
g^{\prime \prime}(t)=s^{\prime}(t) \mathcal{E}_{k}^{\prime}(s(t)(\omega+t p)) \cdot p+s(t) \mathcal{E}_{k}^{\prime \prime}(s(t)(\omega+t p))\left[p, s^{\prime}(t)(\omega+t p)+p\right] .
$$

From $s(0)=1$ and $\mathcal{E}_{k}^{\prime}(\omega)=0$ we get

$$
g^{\prime \prime}(0)=\beta \mathcal{E}_{k}^{\prime \prime}(\omega)[p, \omega]+\mathcal{E}_{k}^{\prime \prime}(\omega)[p, p],
$$

where we have set $\beta=s^{\prime}(0)$. To compute $\beta$ we differentiate (3.6) with respect to $t$ :

$$
\mathcal{E}_{k}^{\prime}(s(t)(\omega+t p)) \cdot p+\mathcal{E}_{k}^{\prime \prime}(s(t)(\omega+t p))\left[\omega+t p, s^{\prime}(t)(\omega+t p)+s(t) p\right]=0 .
$$

Thus at $t=0$ it holds that $\mathcal{E}_{k}^{\prime \prime}(\omega)[\omega, \beta \omega+p]=0$, compared with (3.7), gives

$$
\begin{aligned}
g^{\prime \prime}(0) & =-\frac{\left(\mathcal{E}_{k}^{\prime \prime}(\omega)[p, \omega]\right)^{2}}{\mathcal{E}_{k}^{\prime \prime}(\omega)[\omega, \omega]}+\mathcal{E}_{k}^{\prime \prime}(\omega)[p, p] \\
& =2 \pi\left[\frac{\left(\int_{0}^{1} A(\omega)(\omega \cdot p)\right)^{2}}{1+\int_{0}^{1} A(\omega)}-\int_{0}^{1} A(\omega)(\omega \cdot p)^{2}\right]
\end{aligned}
$$

by (3.3), (3.4) and (3.5). Since 0 is a minimum point for $g$, then $g^{\prime \prime}(0) \geq 0$; that is,

$$
\begin{aligned}
\left(\int_{0}^{1} A(\omega)(\omega \cdot p)^{2}\right)\left(1+\int_{0}^{1} A(\omega)\right) & \leq\left(\int_{0}^{1} A(\omega)(\omega \cdot p)\right)^{2} \\
& \leq\left(\int_{0}^{1} A(\omega)(\omega \cdot p)^{2}\right)\left(\int_{0}^{1} A(\omega)\right)
\end{aligned}
$$

by the Hölder inequality. We infer that $\int A(\omega)(\omega \cdot p)^{2}=0$ for any $p \in \mathbb{C}$, since $A(\omega) \geq 0$ by assumption. Take $p=1 \in \mathbb{C}$ and then $p=i$ to get

$$
0=\int_{0}^{1} A(\omega)\left[(\omega \cdot 1)^{2}+(\omega \cdot i)^{2}\right]=\int_{0}^{1} A(\omega) .
$$

Therefore $A(\omega) \equiv 0$, and the theorem is proved.

From now on we restrict our attention to radially symmetric curvatures $k(P) \equiv$ $k(|P|)$. Quite trivially, if $k$ is any continuous radially symmetric map on $\mathbb{C}$ such that $k(z) \rightarrow k_{\infty}>0$ as $|z| \rightarrow \infty$, then there exists $R>0$ such that $R k(R)=1$. In this case the circle $C_{R}$ is a $k$-loop. In addition, if $u$ is a $k$-loop, then $\mathcal{R} \circ u$ is 
a $k$-loop for any rotation $\mathcal{R}$ of the complex plane. Therefore, the next multiplicity result immediately follows from Theorem 0.1 .

Corollary 3.2. Let $k \in C^{2}(\mathbb{C})$ be a radially symmetric function satisfying $\left(\mathrm{k}_{1}\right)$, $\left(\mathrm{k}_{2}\right)$. Assume that $k^{\prime}(R)>0$ for any $R$ such that $R k(R)=1$. If $\underline{c}$ is achieved, then no minimal $k$-loop is a circle around the origin. Hence there exist at least one round $k$-loop and a rotationally-invariant family of non-round $k$-loops.

It has to be noticed that the class of curvatures described in Corollary 3.2 is non-empty. For instance, let $\lambda>0$ and put

$$
k(r)=1+\lambda \frac{r^{2}-1}{r^{4}+1} .
$$

If $\lambda$ is sufficiently close to 0 , then $k$ satisfies $\left(\mathrm{k}_{1}\right)$ and $\left(\mathrm{k}_{2}\right)$. In addition $R k(R)=1$ if and only if $R=1$, and $k^{\prime}(1)>0$. The existence of a minimal $k$-loop is given by Theorem 2.5,

Remark 3.3. Let $k$ be any continuous radially symmetric curvature. Then the existence of a round $k$-loop centered at 0 is a necessary condition for the existence of $k$-loops. Assume that $g: \mathbb{R} \rightarrow \mathbb{C}$ is a periodic parametrization by arclength of a $k$-loop. Then $g$ solves

$$
\left\{\begin{array}{l}
g^{\prime \prime}=k(|g|)\left(i g^{\prime}\right), \\
\left|g^{\prime}\right|=1 .
\end{array}\right.
$$

Let $P_{0}=g\left(t_{0}\right)$ be a point on $g$ such that $\left|P_{0}\right|=\bar{R}:=\max \{|P| \mid P \in g\}$. Set $v(t):=|g(t)|^{2}$ and compute $v^{\prime \prime}=2\left(\left|g^{\prime}\right|^{2}+g \cdot g^{\prime \prime}\right) \geq 2(1-|k(|g|) g|)$. Since $v$ takes its maximum value at $t_{0}$, then $0 \geq v^{\prime \prime}\left(t_{0}\right) \geq 2(1-|k(\bar{R})| \bar{R})$. Hence $\bar{R}|k(\bar{R})| \geq 1$. By the continuity of the function $r \mapsto r|k(r)|$, there exists a radius $R \in(0, \bar{R}]$ such that $R|k(R)|=1$. Hence the circle $C_{R}$ is a $k$-loop.

Remark 3.4. As an immediate consequence of Remark 3.3 we notice that no $k$ loops may exist if $k: \mathbb{C} \rightarrow \mathbb{R}$ is a continuous radially symmetric function such that $R|k(R)|<1$ for any $R>0$.

\section{UNIQUENESS UP TO SIMILARITY}

In this section we prove Theorem 0.2. We start by fixing some notation. Let $g$ be an embedded loop in $\mathbb{C}$, and let $k: g \rightarrow \mathbb{R}$ be its curvature. We assume that $g$ is positively oriented, so that the interior is to the left. Let $P_{1}, P_{2}$ be two distinct points in $g$. We denote by $A_{g}\left(P_{1}, P_{2}\right)$ the closed arc of $g$ having endpoints $P_{1}$ and $P_{2}$, oriented accordingly to the orientation of $g$. The open arc will be denoted by $\stackrel{\circ}{A_{g}}\left(P_{1}, P_{2}\right)$.

Following Osserman's definition in [10, we let $C_{R}^{g}(X)=\{P \in \mathbb{C}|| P-X \mid=R\}$ be the circumscribed circle about $g$. Thus, $R$ is the minimum positive number $r$ such that there exists a circle of radius $r$ including $g$.

To prove Theorem 0.2 we need a preliminary result, which is based on Hopf's maximum principle. More precisely, the next lemma is a consequence of the Touching Lemma for the mean curvature operator. Although it is essentially well known, we state it here for the sake of completeness.

Lemma 4.1. Let $P \in g \cap C_{R}^{g}(X)$. If $k(M) \leq 1 / R$ for every $M \in g$ close to $P$, then $g \cap C_{R}^{g}(X)$ contains an arc around $P$. 
Proof. Up to a rotation and a translation we can assume that $X=0$ and $P=i R$. An $\operatorname{arc}$ of $C_{R}(0)$ around $P$ is the graph of the function $\gamma(t)=\sqrt{R^{2}-t^{2}}$. Since $g$ is an immersion, then $g$ is locally the graph of a function $f: I=(-\delta, \delta) \rightarrow \mathbb{R}$ such that $f(0)=R$. By assumption $k \leq 1 / R$ in a neighborhood of $P$, and therefore

$$
\left(\frac{\gamma^{\prime}}{\sqrt{1+\left|\gamma^{\prime}\right|^{2}}}\right)^{\prime}+\frac{1}{R}=0 \leq\left(\frac{f^{\prime}}{\sqrt{1+\left|f^{\prime}\right|^{2}}}\right)^{\prime}+\frac{1}{R}
$$

for any $t$ close to 0 . In addition we have that $f \leq \gamma$ and $f(0)=\gamma(0)=R$. Thus $f \equiv \gamma$ in a neighborhood of 0 by Theorem 2.3 in [11].

Proof of Theorem 0.2 . Let $k$ and $g$ be as in the statement of the theorem. Then $g$ is convex, since its curvature is positive. Let $C_{R}^{g}(X)$ be the circumscribed circle about $g$. By Lemma 3 in [10] it turns out that $k(P) \geq 1 / R$ for any $P \in g \cap C_{R}^{g}(X)$.

We claim that $g$ coincides with its circumscribed circle. By contradiction, we assume that $g \cap C_{R}^{g}(X)$ is strictly contained in $C_{R}^{g}(X)$. We distinguish two cases, depending on the number of connected components in $C_{R}^{g}(X) \backslash g$.

Case 1 . The set $C_{R}^{g}(X) \backslash g$ is not connected. Then we can fix two distinct points $P_{1}$ and $P_{2}$ on $g \cap C_{R}^{g}(X)$ such that the $\operatorname{arcs} A_{g}\left(P_{1}, P_{2}\right)$ and $A_{g}\left(P_{2}, P_{1}\right)$ are not contained in the circle $C_{R}^{g}(X)$. By Lemma 4 in [10], there exist two points, $Q_{1} \in \AA_{g}\left(P_{1}, P_{2}\right)$ and $Q_{2} \in \AA_{g}^{\circ}\left(P_{2}, P_{1}\right)$, such that

$$
\left|Q_{i}-X\right|<R=\left|P_{j}-X\right|, \quad k\left(Q_{i}\right)<1 / R \leq k\left(P_{j}\right),
$$

for $i, j=1,2$. Since $k$ is a non-increasing function of the distance from the origin, then $\left|Q_{i}\right|>\left|P_{j}\right|$. Thus $X \neq 0$ and

$$
0<\left|Q_{i}\right|^{2}-\left|P_{j}\right|^{2}=\left|Q_{i}-X\right|^{2}-\left|P_{j}-X\right|^{2}+2 X \cdot\left(Q_{i}-P_{j}\right)<2 X \cdot\left(Q_{i}-P_{j}\right) ;
$$

that is,

$$
\min \left\{X \cdot Q_{1}, X \cdot Q_{2}\right\}>\max \left\{X \cdot P_{1}, X \cdot P_{2}\right\} .
$$

Denote by $\left[Q_{1}, Q_{2}\right]$ the segment joining $Q_{1}$ and $Q_{2}$, and by $\left[P_{1}, P_{2}\right]$ the segment joining $P_{1}$ and $P_{2}$. By the convexity of the curve $g$ the segments $\left[Q_{1}, Q_{2}\right]$ and $\left[P_{1}, P_{2}\right]$ intersect at a point $Q$. Since $Q \in\left[Q_{1}, Q_{2}\right]$, then $X \cdot Q>\max \left\{X \cdot P_{1}, X \cdot P_{2}\right\}$ by (4.1). On the other hand, $X \cdot Q \leq \max \left\{X \cdot P_{1}, X \cdot P_{2}\right\}$ as $Q \in\left[P_{1}, P_{2}\right]$, a contradiction. Thus Case 1 is excluded.

Case 2. The set $C_{R}^{g}(X) \backslash g$ is connected. We find $P_{1}, P_{2} \in g$ such that

$$
C_{R}^{g}(X) \backslash g=\AA_{g}\left(P_{2}, P_{1}\right)
$$

and such that the $\operatorname{arc} A_{g}\left(P_{1}, P_{2}\right)$ is contained in $C_{R}^{g}(X)$. In particular $k(P)=1 / R$ for any $P \in A_{g}\left(P_{1}, P_{2}\right)$. Since $C_{R}^{g}(X)$ is the smallest circle circumscribing $g$, then $A_{g}\left(P_{1}, P_{2}\right)$ is larger than a semicircle ([10], Lemma 2$)$. In particular the antipodal $\tilde{P}_{1}=2 X-P_{1}$ to $P_{1}$ belongs to $A_{g}\left(P_{1}, P_{2}\right)$. Up to a rotation and up to a change of indexes we can assume that $P_{1}, \tilde{P}_{1}$ lie on the same vertical line $\mathcal{V}$, with $P_{1}$ above $\tilde{P}_{1}$ and with $A_{g}\left(P_{1}, \tilde{P}_{1}\right)$ on the left of $\mathcal{V}$.

By Lemma 4 in [10], there exists a point $Q \in \AA_{g}\left(P_{2}, P_{1}\right)$ such that $k(Q)<1 / R$. Since $g$ is convex, then $Q$ lies on the right of the vertical line $\mathcal{V}$. By Lemma 4.1 there exists $M \in A_{g}\left(Q, P_{1}\right)$ on the right of $\mathcal{V}$ such that $k(M)>1 / R$. Since $k \equiv 1 / R$ 
on $A_{g}\left(P_{1}, P_{2}\right)$ and since $k$ is radially symmetric and non-increasing by assumption, then

$$
|M|<|P|<|Q| \text { for any } P \in A_{g}\left(P_{1}, P_{2}\right) \subset C_{R}^{g}(X) \text {. }
$$

In particular, $X \neq 0$, as $|X-Q|<R$. We put $P_{0}:=X\left(1-R|X|^{-1}\right) \in C_{R}^{g}(X)$ and $\tilde{P}_{0}:=2 X-P_{0} \in C_{R}^{g}(X)$.

We claim that 0 cannot belong to $\mathbb{C} \backslash \overline{D_{R}(X)}$. Indeed, if $|X| \geq R$, then the point $P_{0}$ is the minimal distance projection of 0 on $\overline{D_{R}(X)}$. Since $|M|<|P|$ for any $P \in A_{g}\left(P_{1}, P_{2}\right)$ and since $M$ is in the interior of $D_{R}(X)$, then

$$
P_{0} \in \stackrel{\circ}{A}_{g}\left(P_{2}, P_{1}\right) \subset \stackrel{\circ}{A}_{g}\left(\tilde{P}_{1}, P_{1}\right) .
$$

But then, $\tilde{P}_{0} \in A_{g}\left(P_{1}, \tilde{P}_{1}\right)$. Thus $\left|\tilde{P}_{0}\right|<|Q|$ by (4.2). However, this is impossible, as $\left|\tilde{P}_{0}\right|=|X|+R$ and $|Q| \leq|Q-X|+|X|<R+|X|$.

Thus $0 \in D_{R}(X)$ and $\left|P_{0}\right|=R-|X|>0$. Since $\left|P_{1}\right|,\left|\tilde{P}_{1}\right|<|Q|$ and since $Q$ lies on the right of the axes $\mathcal{V}$ joining $P_{1}, \tilde{P}_{1}$, then 0 is on the left of $\mathcal{V}$. Therefore $P_{0} \in A_{g}\left(P_{1}, \tilde{P}_{1}\right) \subset C_{R}^{g}(X)$ and $k\left(P_{0}\right)=1 / R$. Thus $k \equiv 1 / R$ on $C_{\left|P_{0}\right|}(0)$ and $k \leq 1 / R$ outside $D_{\left|P_{0}\right|}(0)$, as $k$ is radially symmetric and non increasing. Finally, we notice that the circle $C_{\left|P_{0}\right|}(0)$ is tangent to $C_{R}^{g}(X)$ at $P_{0}$ from the interior. Thus $k \leq 1 / R$ in a neighborhood of $P_{1}$, and therefore $P_{1}$ is in the interior of $g \cap C_{R}^{g}(X)$ by Lemma 4.1, a contradiction. The theorem is completely proved.

Theorem 0.2 is sharp in view of the next example.

Example 4.2. There exist a positive, radially symmetric and increasing curvature $k: \mathbb{C} \rightarrow \mathbb{R}$ that has a round $k$-loop and a non-round embedded $k$-loop.

To exhibit such a curvature we fix a pair of positive numbers $a<b$. Let $k_{a, b}$ be any smooth function such that

$$
k_{a, b}(z):=\frac{a b}{\left(a^{2}+b^{2}-|z|^{2}\right)^{3 / 2}}
$$

on the disk $\{|z| \leq b\}$. Notice that $k_{a, b}$ can be taken to be radially symmetric and strictly increasing as a function of the distance from the origin.

The ellipse $E_{a, b}$ of equation $a^{-2} x^{2}+b^{-2} y^{2}=1$ has curvature $k$ at any point. For the proof, it is convenient to parametrize $E_{a, b}$ by $g(t)=(a \cos t, b \sin t)$.

By elementary continuity arguments there exists a radius $R \in(a, b)$ such that $R k_{a, b}(z) \equiv 1$ on the circle $C_{R}$, coherently with Remark 3.3 . Thus the circle $C_{R}$ and the ellipse $E_{a, b}$ are distinct $k$-loops.

Theorem 0.2 provides a new characterization of circles.

Corollary 4.3. Round circles are the only convex loops in $\mathbb{C}$ whose curvature is a non increasing function of the Euclidean distance from a fixed point.

We conclude the paper by pointing out the following uniqueness (up to homothety) result, which is an immediate consequence of Theorem 0.2 .

Corollary 4.4. Let $k$ be a continuous, positive and radially symmetric function. If $k$ is radially decreasing, then any embedded $k$-loop is a circle around the origin. 


\section{ACKNOWLedGement}

The author thanks the referee for a careful reading of the manuscript and for valuable comments.

\section{REFERENCES}

[1] Aeppli, A., On the uniqueness of compact solutions for certain elliptic differential equations, Proc. Amer. Math. Soc., 11, 826-832 (1960). MR0121567 (22:12304)

[2] Alexandrov, A.D., Uniqueness theorems for surfaces in the large. I, Vestink Leningrad Univ., 11, 5-17 (1956). Amer. Math. Soc. Transl. Ser. 2, 21, 341-354 (1962). MR0150706(27:698a)

[3] Brezis, H., Coron, J. M., Convergence of solutions of H-systems or how to blow bubbles, Arch. Rat. Mech. Anal., 89, 21-56 (1985). MR784102 (86g:53007)

[4] Caldiroli, P., Guida, M., Closed curves in $\mathbb{R}^{3}$ with prescribed curvature and torsion in perturbative cases. I. Necessary condition and study of the unperturbed problem, Atti Accad. Naz. Lincei Cl. Sci. Fis. Mat. Natur. Rend. Lincei (9) Mat. Appl., 17, 227-242 (2006). MR:2254070 (2007f:53004)

[5] Caldiroli, P., Guida, M., Helicoidal trajectories of a charge in a nonconstant magnetic field, Adv. Differential Equations, 12, 601-622 (2007). MR2319450(2008j:58013)

[6] Caldiroli, P., Musina, R., Existence of minimal H-bubbles, Commun. Contemp. Math., 4, 177-209 (2002). MR1901145 (2004b:53013)

[7] Caldiroli, P., Musina, R., Bubbles with prescribed mean curvature: the variational approach, Nonlinear Analysis TMA, to appear, DOI:10.1016/j.na.2011.01.019

[8] Guida, M., Perturbative-type results for some problems of geometric analysis in low dimension, Ph.D. Thesis, Università di Torino (2004).

[9] Guida, M., Rolando, S., Symmetric k-loops, Differential Integral Equations, 23, 861-898 (2010). MR 2675586

[10] Osserman, R., The four or more vertex theorem, Amer. Math. Monthly, 92, 332-337 (1985). MR.790188 (87e:53001)

[11] Pucci, P., Serrin, J., The strong maximum principle revisited, J. Diff. Equations, 196, 1-66 (2004). MR 2025185 (2004k:35033)

[12] Schneider, M., Multiple solutions for the planar Plateau problem, preprint, arXiv:0903.1132 (2009).

[13] Treibergs, A.E., Wei, W., Embedded hyperspheres with prescribed mean curvature, J. Differential Geom., 18, 513-521 (1983). MR.723815(85e:53082)

Dipartimento di Matematica ed Informatica, Universitì di Udine, via delle Scienze, 206-33100 Udine, ITALY

E-mail address: roberta.musina@uniud.it 